\title{
Low-temperature magnetic ordering and structural distortions in Vanadium Sesquioxide $\left(\mathrm{V}_{2} \mathrm{O}_{3}\right)$
}

\author{
Daniel Grieger and Michele Fabrizio \\ SISSA, Via Bonomea 265, 34136 Trieste, Italy
}

\begin{abstract}
Vanadium Sesquioxide $\left(\mathrm{V}_{2} \mathrm{O}_{3}\right)$ is an antiferromagnetic insulator below $T_{\mathrm{N}} \approx 155 \mathrm{~K}$. The magnetic order is not of C- or G-type as one would infer from the bipartite character of the hexagonal basal plane in the high-temperature corundum structure. In fact, the Néel transition is accompanied by a monoclinic distortion that makes one bond of the honeycomb plane inequivalent from the other two, thus justifying a magnetic structure with one ferromagnetic bond and two antiferromagnetic ones. We show here that the magnetic ordering, the accompanying monoclinic structural distortion, the magnetic anisotropy and also the recently discovered high-pressure monoclinic phase, can all be accurately described by conventional electronic structure calculations within GGA and GGA+U. Our results are in line with DMFT calculations for the paramagnetic phase $e^{\underline{1}}$, which predict that the insulating character is driven by a correlation-enhanced crystal field splitting between $e_{g}^{\pi}$ and $a_{1 g}$ orbitals that pushes the latter above the chemical potential. We find that the $a_{1 g}$ orbital, although almost empty in the insulating phase, is actually responsible for the unusual magnetic order as it leads to magnetic frustration whose effect is similar to a next-nearest-neighbor exchange in a Heisenberg model on a honeycomb lattice.
\end{abstract}

PACS numbers: 71.45.Gm, 71.45.Lr, 71.30.+h, 73.20.Mf, 71.15.Mb

\section{INTRODUCTION}

For more than forty years, the phase diagram of Chromium/Titanium-doped Vanadium Sesquioxide 2,3 $\left(\mathrm{V}_{2} \mathrm{O}_{3}\right)$ has gathered great interest, especially because of its isostructural high-temperature paramagnetic metal to paramagnetic insulator transition, which is by now considered the prototypical realization of a genuine Mott transition, i.e. not corrupted by any symmetry breaking. Relatively less attention has instead been paid on the low temperature antiferromagnetic phase of $\mathrm{V}_{2} \mathrm{O}_{3}$. Indeed, within a certain doping/pressure range, Vanadium Sesquioxide undergoes a magnetic phase transition $\underline{4}$ below a critical Néel temperature $T_{\mathrm{N}}, \stackrel{4}{\underline{-6}}$ which is around $155 \mathrm{~K}$ for undoped $\mathrm{V}_{2} \mathrm{O}_{3} \stackrel{7}{*}$ Since magnetism in a stronglycorrelated material is just a side effect of Mott's localization, scientific interest in $\mathrm{V}_{2} \mathrm{O}_{3}$ has mostly focused so far on the latter phenomenon rather than on the mechanism that produces the experimentally observed magnetic order. Indeed, the magnetic structure in $\mathrm{V}_{2} \mathrm{O}_{3}$ rises a number of intriguing questions most of which are still awaiting an answer.

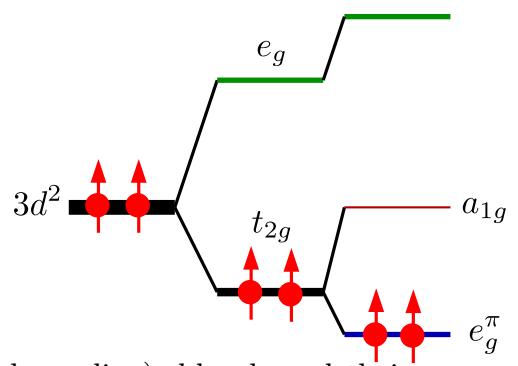

FIG. 1: (Color online) $d$ levels and their occupancy of a hypothetical isolated Vanadium atom in the trigonal field of the high-temperature corundum structure.
In $\mathrm{V}_{2} \mathrm{O}_{3}$, each Vanadium atom has two electrons within the $t_{2 g}$ orbitals of the cubic-split $d$-shell, as schematically shown in Fig. 1, In the high-temperature corundum structure, the trigonal field further splits the $t_{2 g}$ into a lower $e_{g}^{\pi}$ doublet and a higher $a_{1 g}$ singlet. In the extreme Mott localized scenario, the two electrons would occupy the $e_{g}^{\pi}$ orbitals and be coupled into a spin $S=1$ configuration in accordance with Hund's rules, see Fig. 1 . This idealized picture, each $e_{g}^{\pi}$ singly occupied and the $a_{1 g}$ empty, is not far from what most recent LDA+DMFT calculations predict. 1,8 If we assume legitimate to discard the $a_{1 g}$ contribution to the low-energy processes that control the coupling between the $S=1$ localized moments, we must conclude that the virtual hopping of $e_{g}^{\pi}$ electrons between nearest neighbor sites gives rise to a conventional antiferromagnetic super-exchange within the honeycomb basal plane, whose bipartite character would then lead to a Néel two-sublattice antiferromagnetism. Moreover, since the $e_{g}^{\pi}$ orbitals are non-bonding along the $c$-axis perpendicular to the hexagonal plane, we would expect an antiferromagnetic order either of the G- or the C-type, which we shall hereafter denote as "simple" $\underline{9}$ and "layered" antiferromagnetic structures, respectively.

In reality, the experimentally observed magnetic structure ${ }^{5}$, which we shall refer to as "true", is completely different. Along the $c$-axis, the two nearest neighbor Vanadium atoms are coupled ferromagnetically, not in disagreement with the above expectation. In contrast, among the three bonds connecting one Vanadium atom to its nearest neighbors within the honeycomb basal plane, only two are antiferromagnetic but the remaining one is ferromagnetic. This phase is accompanied by a monoclinic distortion which goes along with the magnetic structure, making one hexagonal bond inequivalent from the other two. However, slightly contradicting results about the exact influence onto the respective bond- 
lengths can be found in literature, from shortening the antiferromagnetic bonds, $\stackrel{7,10}{=}$ as one would reasonably expect, to the opposite $\underline{11}$

The natural issue that arises is why this complicated "true" magnetic order should be energetically favorable with respect to the "simple" or "layered" structures in view of the additional energy cost of the monoclinic distortion. Up to now, this simple question has still not found a satisfactory answer.

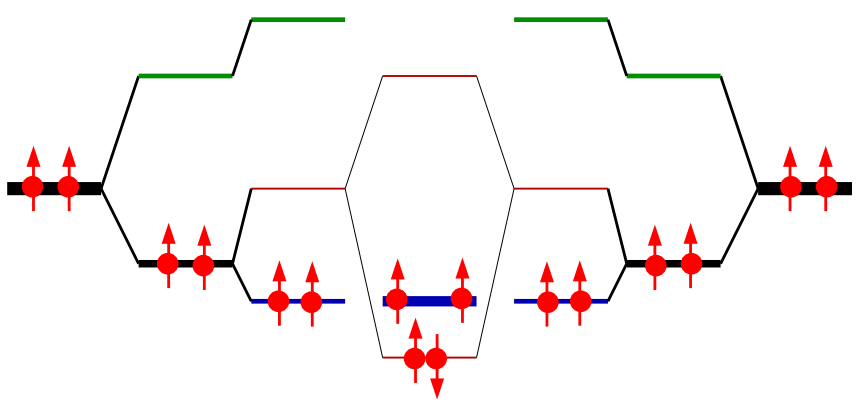

FIG. 2: (Color online) The "dimer" building block of Castellani, Natoli and Ranninger, with its electronic configuration.

The first attempt to explain the observed magnetic structure was performed in a series of papers by Castellani, Natoli and Ranninger $(\mathrm{CN \& R}) \stackrel{12-14}{\underline{14}}$ Their starting point was not the atomic limit of Fig. 1, but the molecule of two nearest neighbor Vanadium atoms along the $c$-axis, which we shall refer to as a "dimer". The $a_{1 g}$ orbitals form a covalent bond along the $c$-axis that falls below the $e_{g}^{\pi}$ levels, see Fig. 2. The lowest electronic configuration consists then of two electrons in a spin-singlet configuration occupying the $\sigma$-bond, and the remaining two coupled into a spin-triplet configuration within the $e_{g}^{\pi}$ levels. The residual fourfold orbital degeneracy besides the threefold spin degeneracy was exploited to build a spin-orbital Kugel'-Komskii ${ }^{15}$ type of Heisenberg model, whose mean-field solution in a certain parameter range reproduces the observed magnetic structure and simultaneously predicts an orbital ordering. The CN\&R's scenario implies that each Vanadium has spin $S=1 / 2$, while the dimer has $S=1$. In order to explain the observed magnetic moment larger than one Bohr magneton, CN\&R invoked an exchange polarization of the $a_{1 g}$ electrons.

The "dimer" building block was later questioned on the basis of x-ray absorption measurements $\frac{16}{}$ and of abinitio LDA $+\mathrm{U}$ calculations,$\frac{9}{,}$ both supporting a scenario in which each Vanadium is in a spin $S=1$ configuration rather than spin- $1 / 2$. This conclusion was further reinforced by x-ray resonant elastic scattering measurements showing that the observed moment of $1.2 \mu_{B}$ has both a spin contribution $2\langle S\rangle \simeq 1.7$ as well as an orbital one $\langle L\rangle \simeq-0.5 \stackrel{17}{\simeq}$ All these novel results stimulated attempts to reexamine the $\mathrm{CN} \& \mathrm{R}$ model in terms of $S=2$ dimers (each Vanadium in a spin-triplet state) rather than $S=1$ as in the original formulation, see e.g. Refs. 18, 19 and 20, although this list is by no means exhaustive.
At the meantime, the same belief that the $\mathrm{V}_{2}$ dimer is the relevant building block to explain the magnetic structure started to be questioned, $\stackrel{21-23}{2}$ until most recent LDA+DMFT calculations 1,8 have finally come back to the atomic scenario of Fig. 1 as the most plausible one for the insulating phases of $\mathrm{V}_{2} \mathrm{O}_{3}$. Nevertheless, important issues remain open, which might escape from very accurate but still approximate techniques like $\mathrm{LDA}+\mathrm{U}$ or LDA+DMFT 23,24

An important one is the aforementioned sizable orbital contribution to the magnetic moment 17 We observe that the $t_{2 g}$ orbitals can make available at most an orbital moment $L=1$. Therefore the observed $|\langle L\rangle| \sim 0.5$ is a substantial part of it, which cannot be justified within the atomic limit of Fig. 1, since the $e_{g}^{\pi}$ alone are not spin-orbit active, but can be explained within the dimer scenario $\stackrel{20,24}{2}$

Equally intriguing remains the monoclinic distortion accompanying the magnetic order. As we mentioned, if we assume the atomic limit of Fig. 1 and neglect contributions from $a_{1 g}$ orbitals, only "simple" or "layered" magnetic structures can be stabilized. More realistic LDA+U calculations by Ezhov et al $\stackrel{9}{\frac{9}{2}}$ show that in the corundum structure the lowest energy magnetic configuration is indeed the "simple" one, lower by $5 \mathrm{~K}$ than the "true" structure. Since the monoclinic distortion costs elastic energy, it is not easy to conceive on the basis of these calculations why $\mathrm{V}_{2} \mathrm{O}_{3}$ would distort to stabilize a phase that in the undistorted crystal is higher in energy. Tight-

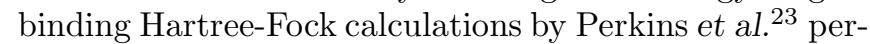
formed with hopping matrix elements of symmetry appropriate to the corundum phase find that the "true" structure can have lower energy than the "simple" one even in the undistorted lattice, though in a quite narrow region of parameter space. Similar conclusions are obtained within the dimer model ${ }^{18,19}$

In summary, in spite of many attempts performed in the last forty years, the real cause of the observed magnetic order and concomitant monoclinic distortion in $\mathrm{V}_{2} \mathrm{O}_{3}$ is still elusive and we believe it is worth trying to shed further light, which we hope to do in the present study. The approach we shall adopt is mainly plain density functional theory (DFT) and its DFT $+\mathrm{U}$ extension to strong electronic correlations, which is especially suitable for antiferromagnetic insulators. In this sense, this work is partially an extension of the pioneering one by Ezhov et $a l^{9} \stackrel{9}{*}$ Furthermore, model studies are shown to support the findings from these realistic theories.

The paper is organized as follows: Section [I summarizes the density functional theory (DFT) picture of $\mathrm{V}_{2} \mathrm{O}_{3}$ regarding magnetism and structural distortions. Section III enhances this picture by strong electronic correlations as described by DFT $+\mathrm{U}$ approaches. SectionIV focuses on the orientation of the magnetic moments as described by DFT $+\mathrm{U}$ as well as by an analytic picture. Section $\mathrm{V}$ finally aims at finding reasons for the type of magnetic ordering realized in $\mathrm{V}_{2} \mathrm{O}_{3}$ by considering suitable model studies. 


\section{DFT STUDIES}

It is obvious that electronic correlations do play a crucial role in the physics of $\mathrm{V}_{2} \mathrm{O}_{3}$, as demonstrated in detail by several earlier studies, $1,8,9,25$ to which the antiferromagnetic ground state makes no exception. However, already density functional theory (DFT) in its generalizedgradient approximation (GGA) to the energy functional (here in its $\mathrm{PBE}$ parametrization ${ }^{26}$ ), in spite of being known to fail for several strongly correlated systems, can give some important insights into this state. It is understood that one cannot expect an accurate description of all the observed properties by plain GGA, but it will be shown to be a useful starting point for all further considerations.

The following DFT calculations have been performed with the QUANTUM ESPRESSO code 27 using ultrasoft pseudopotentials (V.pbe-n-van.UPF and O.pbevan_ak.UPF from http://www.quantum-espresso.org). To account for the monoclinic distortion and magnetic ordering, a supercell containing 8 Vanadium atoms is used. Its symmetry properties are described by the monoclinic space group $I 2 / a, \stackrel{7,28}{,}$ whose lattice vectors $\left(\mathbf{a}_{m}, \mathbf{b}_{m}, \mathbf{c}_{m}\right)$ can be built from the original high-temperature corundum structure lattice vectors $\left(\mathbf{a}_{\mathrm{H}}, \mathbf{b}_{\mathrm{H}}, \mathbf{c}_{\mathrm{H}}\right)$ in hexagonal notation as follows:

$$
\left(\begin{array}{c}
\mathbf{a}_{m} \\
\mathbf{b}_{m} \\
\mathbf{c}_{m}
\end{array}\right)=\left(\begin{array}{ccc}
\frac{2}{3} & \frac{4}{3} & \frac{1}{3} \\
1 & 0 & 0 \\
\frac{1}{3} & \frac{2}{3} & -\frac{1}{3}
\end{array}\right)\left(\begin{array}{c}
\mathbf{a}_{\mathrm{H}} \\
\mathbf{b}_{\mathrm{H}} \\
\mathbf{c}_{\mathrm{H}}
\end{array}\right) .
$$

If not stated otherwise, the length of the unit vectors will not be altered throughout the following calculations, but retained at its experimentally reported value at ambient conditions $^{28}$.

\section{A. The paramagnetic solution}

The most basic GGA setup that can be built for $\mathrm{V}_{2} \mathrm{O}_{3}$ is a calculation with enforced paramagnetism. If done in the enlarged 8-site unit cell and allowing for relaxation of the atomic positions, its solution has the noteworthy peculiarity that it incorporates a monoclinic distortion. In spite of the elastic energy cost that is associated to any kind of lattice distortion, the energy gain compared to the relaxed paramagnetic corundum structure is as large as $25 \mathrm{meV}$ per Vanadium atom. The distortion is characterised by two nearest-neighbor bonds being lengthened and one shortened in the hexagonal $a_{\mathrm{H}} b_{\mathrm{H}}$ plane. Anticipating results of the next section, we mention that the same kind of distortion occurs in the antiferromagnetic metal phase. However, if GGA is supplemented by a Hubbard $U$, above a threshold value an antiferromagnetic insulating phase is established, which still has monoclinic distortion but with two bonds shortened and one lengthened, hence opposite to that in the metal phases, either paramagnetic or antiferromagnetic.
The large energy gain corresponds to a substantial lattice distortion, with the length of the shortened bond in the $a_{\mathrm{H}} b_{\mathrm{H}}$ plane of $2.56 \AA$ and thus even slightly smaller than that of the Vanadium dimer along the $c_{\mathrm{H}}$ direction of $2.63 \AA$. Comparing with the length of the enlarged bonds in the $a_{\mathrm{H}} b_{\mathrm{H}}$ plane of about $3.0 \AA$, one notices that the structure becomes similar to an array of 1d-chains, each of them running along the dimer and the short bond in the hexagonal plane.

Although such a monoclinic paramagnetic metal phase is unstable against magnetism, as we shall show in the next section, it is nevertheless of interest in view of the recent discovery of a high-pressure monoclinic metal phase $^{29}$ that is actually not dissimilar to what we just found. It is therefore worth investigating the mechanisms that may drive such a distortion. (a)

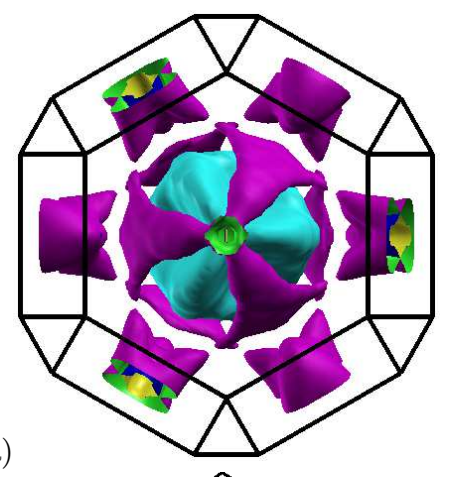

(b)

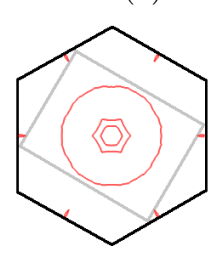

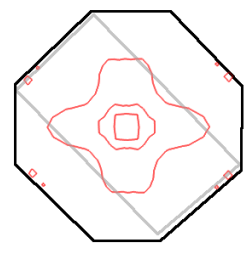

FIG. 3: (Color online) (a) Fermi surface of paramagnetic metallic corundum structure $\mathrm{V}_{2} \mathrm{O}_{3}$ with experimental atomic positions ${ }^{28}$, seen along the crystallographic $c_{\mathrm{H}}$ direction, equivalently the cartesian $z$ axis. (b) Projections of the Fermi surface onto planes that are obtained by rotating around the cartesian $y$-axis of panel (a). While the vertical axis corresponds to the cartesian $y$ direction, the horizontal axis is: the cartesian $x$ direction (left panel); rotated about $40^{\circ}$ with respect to the former, so that it represents the direction towards a next-nearest neighbour atom in the adjacent hexagonal plane (middle panel); perpendicular to the latter (right panel). The black lines indicate the Brillouin zone boundary of the corundum structure, the grey lines its shape for the doubled monoclinic cell.

We believe that the monoclinic distortion is actually driven by a Fermi surface nesting of the corundum band structure. Indeed the central sheet of the whole Fermi surface, shown in Fig. 3(a) and calculated with the structural data of Ref. 28 corresponding to the hightemperature paramagnetic metal, has nesting properties compatible with an instability towards a monoclinic distortion with a unit cell doubling. This is quite evident 
by looking at cut planes as shown in Fig. [3(b). While the aforementioned sheet looks almost circular in the honeycomb plane of Vanadium atoms, relatively large regions of parallel surfaces can be identified by slightly tilting the cut-plane. In order to better uncover the driving physical

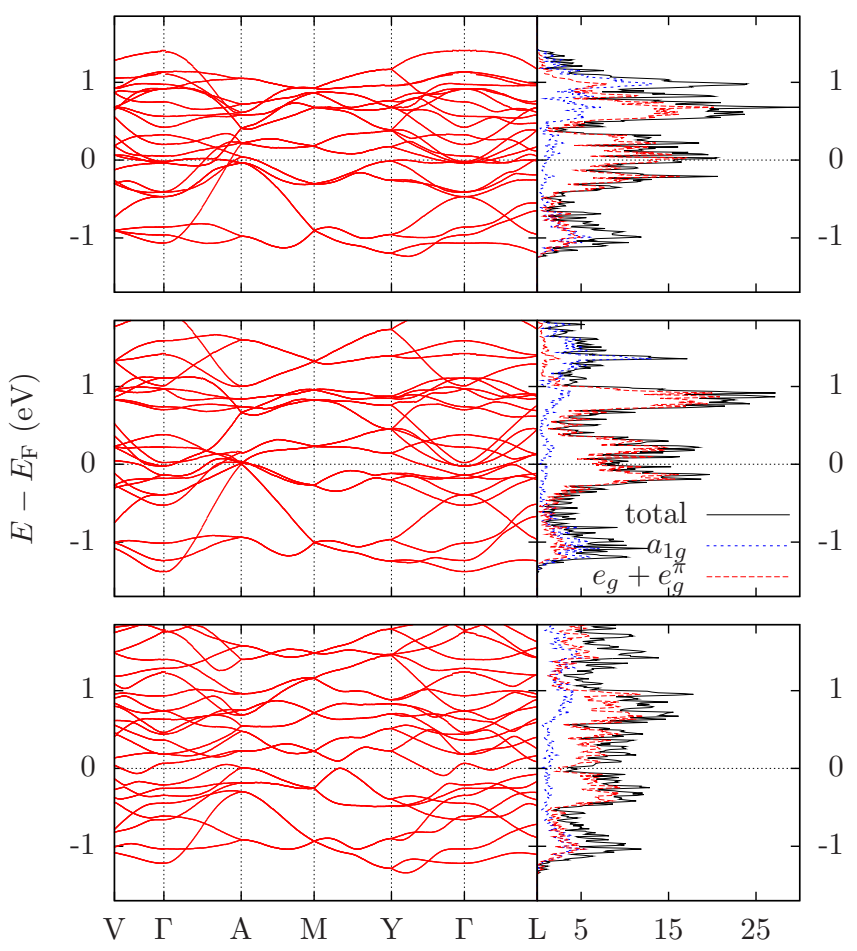

FIG. 4: (Color online) Band structure (left) and projected density of states (right) from GGA of paramagnetic corundum structure $\mathrm{V}_{2} \mathrm{O}_{3}$ with atomic positions determined experimentally for the paramagnetic metallic phase ${ }^{28}$ (up), GGArelaxed atomic positions in the corundum structure (middle) and GGA-relaxed atomic positions allowing for a monoclinic distortion (down).

mechanism, the band structures in the enlarged 8-atom unit cell shown in Fig. 4 are particularly enlightening. The chosen path through the Brillouin zone pertaining to the enlarged 8-atom monoclinic cell starts from the point $\mathrm{V}=\left(0, \frac{1}{4},-\frac{1}{4}\right)$ in relative reciprocal coordinates (compatible with the magnetic order), moves further to $\Gamma$ and along the Vanadium dimer ( $\Gamma$-A direction), further goes in the plane perpendicular thereto at the "upper" $\left(k_{z}=\frac{1}{2}\right)$ edge of the Brillouin zone $(\mathrm{A}-\mathrm{M})$, back to the original hexagonal $a_{\mathrm{H}} b_{\mathrm{H}}$ plane at $k_{z}=0(\mathrm{M}-\mathrm{Y})$ and continues in this plane (Y- $\Gamma-\mathrm{L})$.

Starting from the well-known ${ }^{30.31}$ GGA low-energy density of states and band structure of the corundum paramagnetic metal, as displayed in the top row of Fig. (4. one immediately notices a large density of states directly at the Fermi energy, due to almost flat bands near $\Gamma$. As expected, relaxation of the atomic positions within the corundum structure (middle row of Fig. (4) partially reduces this instability, and furthermore leads to a reduction of the splitting between $e_{g}^{\pi}$ and high-energy $e_{g} \cdot \underline{32}$
However, allowing for a monoclinic distortion (bottom row of Fig. (4) further stabilizes the system by splitting the flat bands around $\Gamma$ and opening a pseudo-gap at the Fermi energy. We mention that the undistorted structure is not even metastable, but corresponds to a saddle point in the total energy.

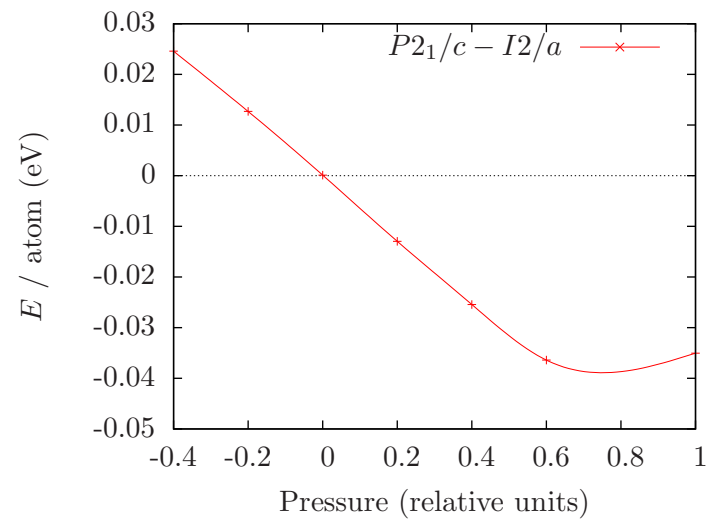

FIG. 5: (Color online) Total energy difference of the $P 2_{1} / c$ and $I 2 / a$ phases of monoclinic $\mathrm{V}_{2} \mathrm{O}_{3}$, as a function of the applied pressure. The relative units of the pressure axis correspond to the reported values in the corundum structure at ambient conditions ${ }^{28}(0.0)$ and the experimentally reported values of the high-pressure phase transition from $I 2 / a$ to $P 2_{1} / c^{29}(1.0)$.

We finally mention that the above monoclinic paramagnetic metal phase is not exactly equivalent to that observed at high-pressure in Ref. 29, which is characterised by a further symmetry lowering from $I 2 / a$ to $P 2_{1} / c$. Figure 5 shows that this symmetry reduction can also be seen in GGA upon simulating pressure by a decrease of the unit cell volume. Both phases $I 2 / a$ and $P 2_{1} / c$ are minima of the total energy. At ambient pressure, $I 2 / a$ has a slightly lower energy than $P 2_{1} / c$, but the situation is reverted already applying small pressure. Therefore the transition from $I 2 / a$ to $P 2_{1} / c$ would occur according to GGA at significantly lower pressures than reported experimentally ${ }^{29}$. We believe that this is an artifact due to GGA underestimation of electronic correlations. Indeed, the $P 2_{1} / c$ structure in GGA is characterised by a charge disproportionation between inequivalent Vanadium atoms, which is hindered by electronic correlations.

\section{B. Magnetic solutions}

Allowing for magnetism in the framework of spinpolarized GGA, Tab. II shows that the paramagnetic GGA solution described in the previous subsection is unstable compared to all of the magnetic solutions. Likewise, the energy gain due to its monoclinic distortion is significantly smaller than the energy gain due to magnetic exchange.

Comparing the different possible magnetic orderings, an important result that we find is the stability of the 
TABLE I: Comparison of some basic quantities, calculated for the different antiferromagnetic configurations of $\mathrm{V}_{2} \mathrm{O}_{3}$ within plain GGA. Energies are given relative to the respective "simple" phase.

\begin{tabular}{lccc}
\hline \hline & "simple" & "true" & "layered" \\
\hline experimental corundum structure & & & -4.1 \\
total GGA energy $(\mathrm{meV} / \mathrm{V}$ atom) & 0 & 1.57 & -5.2 \\
absolute magnetization $\left(\mu_{\mathrm{B}} / \mathrm{V}\right.$ atom) & 1.53 & & 1.54 \\
\hline relaxed structures & & -12.2 & 0 \\
total GGA energy $(\mathrm{meV} / \mathrm{V}$ atom $)$ & 0 & 1.55 & -7.9 \\
absolute magnetization $\left(\mu_{\mathrm{B}} / \mathrm{V}\right.$ atom) & 1.47 & 1.49 \\
\hline \hline
\end{tabular}

"true" antiferromagnetic ordering already in GGA. Note that this requires relaxation of the atomic positions within the crystal cell. Also this relaxation of the "true" antiferromagnetic structure reveals a monoclinic distortion similar to the experimentally observed one and similar (but smaller) to the one described above for the paramagnetic regime. Note that both the other two investigated antiferromagnetic configurations ("layered" and "simple") remain in an undistorted corundum structure even allowing for structural relaxation. This can also be expected by symmetry considerations of the magnetic structure, recalling that the magnetic exchange energy gain is significantly larger than an energy gain of the structural distortion. This underlines the fact that the lattice distortion in antiferromagnetic $\mathrm{V}_{2} \mathrm{O}_{3}$ directly stabilizes the antiferromagnetic order, and is thus closely related to it.

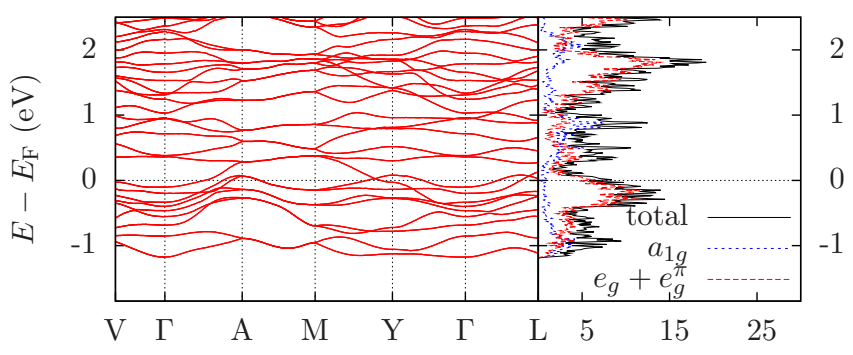

FIG. 6: (Color online) Band structure (left) and projected density of states (right) of true antiferromagnetic $\mathrm{V}_{2} \mathrm{O}_{3}$ from GGA.

As can be seen from the low-energy density of states and band structure shown in Fig. 6, the main drawback is that pure GGA cannot describe the insulating behavior of the magnetic structure. This is the expected result in view of the importance of strong electronic correlations in $\mathrm{V}_{2} \mathrm{O}_{3}$. Furthermore, one notices that the splitting between $a_{1 g}$ and $e_{g}^{\pi}$ turns out to be small enough that all orbitals from the $t_{2 g}$ block have a similar filling (with the $a_{1 g}$ occupation slightly smaller than each $e_{g}^{\pi}$ ). This gives rise to a spin magnetic moment slightly smaller than the observed experimental value, which we recall is $\sim 1.7 \mu_{B}$ once corrected by the orbital contribution.

\section{DFT+U STUDIES}

Since the antiferromagnetic insulating state is the ground state of $\mathrm{V}_{2} \mathrm{O}_{3}$, one can expect to gain insight into the effects of strong electronic correlations already from a simple method like GGA+U. Here, the simplified version of Cococcioni and de Gironcoli 33 in the QUANTUM ESPRESSO package is put into practice, which implies the use of only one effective parameter $U-J$ and a fully-localized limit (FLL) double-counting correction.

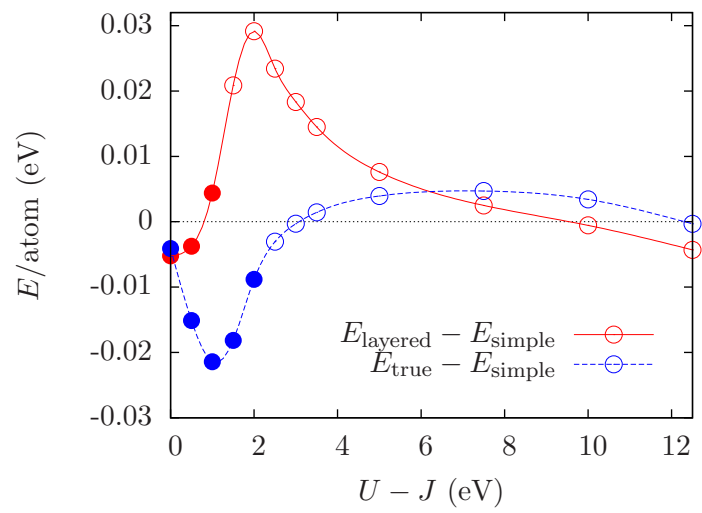

FIG. 7: (Color online) Phase stability comparison in the experimentally determined corundum structure of $\mathrm{V}_{2} \mathrm{O}_{3}$ within GGA+U. Closed circles indicate metallic, open circles indicate insulating solutions.

Since the actual value of the parameter $U-J$ is a priori unknown, Fig. 7 compares the stability of each of the possible magnetic structures in terms of their total energy for a wide parameter range. This calculation is performed in the experimental atomic positions in the unrelaxed corundum structure, $\stackrel{28}{,}$ nevertheless already shows that a small $U-J$ is able to stabilize the "true" antiferromagnetic phase, whereas for large interactions the "simple" and "layered" structures become more stable.

The relaxation of the structural parameters within GGA+U further stabilizes the "true" antiferromagnetically ordered phase and extends its stability region, as shown in Fig. 8. As anticipated, above $U-J \simeq 2.0 \mathrm{eV}$, the "true" antiferromagnetic state turns from metallic into insulating, which sets between 2.0 and $4.0 \mathrm{eV}$ the range of $U-J$ values that reproduce within $\mathrm{GGA}+\mathrm{U}$ 


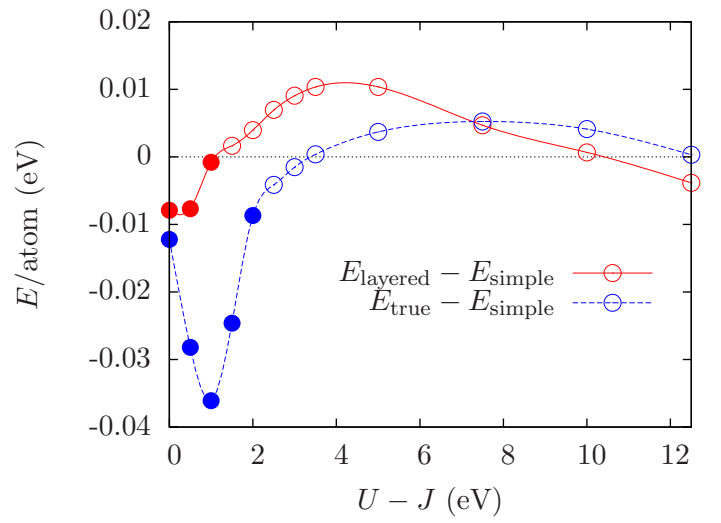

FIG. 8: (Color online) Phase stability comparison in the relaxed structure of $\mathrm{V}_{2} \mathrm{O}_{3}$ within $\mathrm{GGA}+\mathrm{U}$. Filled circles indicate metallic solutions of the layered/true phase, open circles indicate insulating solutions thereof.

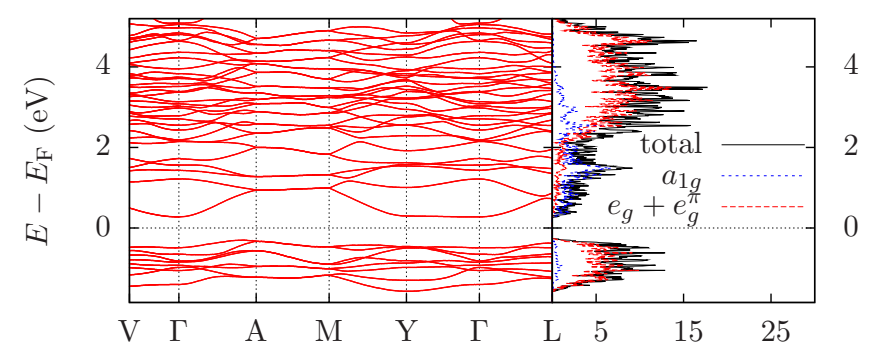

FIG. 9: (Color online) Band structure (left) and projected density of states (right) of true antiferromagnetic $\mathrm{V}_{2} \mathrm{O}_{3}$ from $\mathrm{GGA}+\mathrm{U}$ at $U-J=3.0 \mathrm{eV}$.

the magnetic and conducting properties of the actual material. Fig. 9 shows the GGA+U Kohn-Sham band structure and density of states projected onto Vanadium $d$-orbitals for $U-J=3.0 \mathrm{eV}$, which we assume to be a realistic estimate.

It turns out that the GGA $+\mathrm{U}$ realization of the insulating phase corresponds to the straightforward solution of occupied $e_{g}^{\pi}$ orbitals and empty $a_{1 g}$ one, thus implying an $S=1$ spin configuration. As mentioned, this is in line with e. g. the $U$-induced paramagnetic Mott metalinsulator transition in DMFT ${ }^{1}$, though still a matter of debate $\stackrel{23,24}{24}^{2}$

This scenario is further confirmed in Fig. 10, where we plot the occupation numbers in the crystal field basis that diagonalizes the GGA+U occupation matrix. We mention that, since these orbitals are not strongly localized on a single Vanadium atom, the total occupation is not precisely 2 . The depletion of the $a_{1}$-like crystal-field basis orbital with increasing values of $U-J$ in favor of $e_{g}^{\pi}$-like orbitals is evident. A noteworthy jump occurs at the metal-insulator transition, with the above-mentioned scenario of a majority-spin $a_{1 g}$ orbital that is almost halffilled below and almost empty above.

One remarkable effect of the monoclinic distortion and the magnetic ordering is the lifting of the degeneracy bewteen the two $e_{g}^{\pi}$ orbitals, which is particularly strong

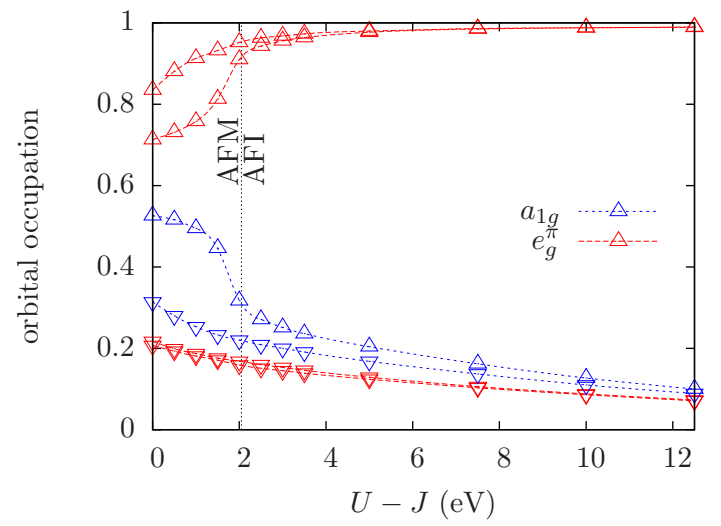

FIG. 10: (Color online) Crystal field basis occupation numbers (eigenvalues of the GGA+U local density matrix) per Vanadium d-orbital as a function of $U-J$ in the relaxed true antiferromagnetic phase of $\mathrm{V}_{2} \mathrm{O}_{3}$. Triangles pointing up indicate the majority spin channel, triangles pointing down the minority spin channel. As before, AFM and AFI stand for antiferromagnetic metal and insulator, respectively.

in the (unphysical) magnetic metallic regime, but still present in the magnetic insulating one. It is a direct consequence of the breaking of the three-fold rotational symmetry of the Vanadium planes with magnetic order or structural distortion, and therefore does not occur for the other investigated magnetic structures that do not break such symmetry.

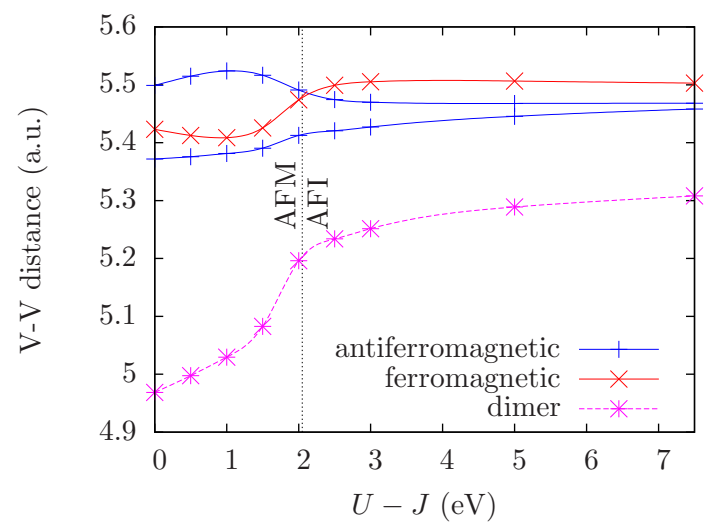

FIG. 11: (Color online) Nearest-neighbour Vanadium distances from relaxation in the "true" antiferromagnetic structure, indicating the monoclinic distortion. AFM and AFI stand for antiferromagnetic metal and insulator, respectively.

The inequality between the two $e_{g}^{\pi}$ orbitals, more accentuated the smaller $U-J$, also shows up into different lengths of the two antiferromagnetic in-plane nearestneighbor bonds, as shown in Fig. 11, to such an extent that, in the metal phase $U-J<2 \mathrm{eV}$, one of the antiferromagnetic bonds is the longest. On the contrary, above $U-J=2 \mathrm{eV}$, i.e. in the realistic insulating phase, all nearest-neighbor Vanadium distances shown in the same Fig. 11 are compatible with the observed monoclinic distortion and with the intuitive expectation 
of elongated ferromagnetic bonds and shortened antiferromagnetic ones. However a small difference between the lengths of the two in-plane antiferromagnetic bonds persists also in the insulating side.

\section{MAGNETIC ANISOTROPY}

Already the original 1970's work by Moon ${ }^{5}$ pointed out that the magnetic moments are oriented with a certain angle towards the crystallographic $c$-direction. Such a magnetic anisotropy is, at first glance, not expected for a light element like Vanadium. However, for similar compounds like Vanadium spinels, values of spinorbit coupling in the range of $13-20 \mathrm{meV}$ have been reported ${ }^{34}-37$, which makes a DFT calculation including relativistic effects (spin-orbit coupling) and noncollinear magnetism worth trying. To this end, the implementation thereof ${ }^{38}$ in QUANTUM ESPRESSO has been used with a fully relativistic pseudopotential (V.rel-pbe-spnl-rrkjus_psl.1.0.0.UPF from the PSLibrary of http://www.quantum-espresso.org) and the rotationally invariant GGA $+\mathrm{U}$ formulation of Liechtenstein et al. ${ }^{39}$. Due to the high computational demands of such a calculation, no further relaxation of the structural parameters has been done, but values of the monoclinically distorted collinear-antiferromagnetic structure relaxed without $U$ have been used.

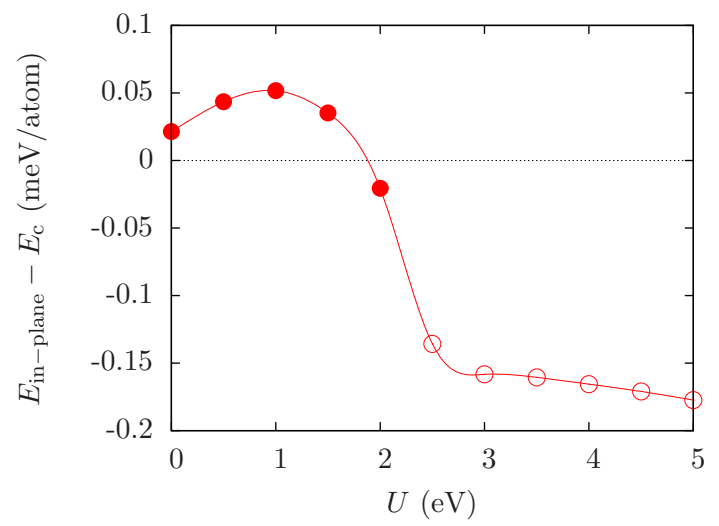

FIG. 12: (Color online) LDA+U energy difference of "true" antiferromagnetic solutions with spin orientation in the honeycomb plane and in $c$-direction (perpendicular thereto), as a function of the Hubbard parameter $U$. Closed circles indicate metallic, open circles indicate insulating solutions.

Fig. 12 shows the energy difference between GGA+U solutions with magnetic moments oriented in the honeycomb plane $\left(E_{\text {in-plane }}\right)$ and oriented perpendicular thereto $\left(E_{\mathrm{c}}\right)$, i. e. in the crystallographic $c$-direction. A first result is that, without electronic correlations, i. e. at the GGA level, the $c$-axis alignment is favored, although with a tiny energy difference. However, upon increasing $U$, and specifically above the metal-insulator transition, the in-plane orientation becomes more stable.
Indeed there is a close connection between the change from easy-axis to easy-plane and the $a_{1 g}$ depletion that occurs at the metal-insulator transition, which can be illustrated by a simple model calculation.

Let us assume an isolated Vanadium in the trigonal crystal field. A suitable basis set for the $t_{2 g}$ manifold (with corundum symmetry) can be written (the sign is related to the multiple Vanadium atoms per unit cell):

$$
\begin{aligned}
& \left|a_{1 g}\right\rangle=\left|d_{3 z^{2}-r^{2}}\right\rangle, \\
& \left|e_{g 1}\right\rangle=\sqrt{\frac{2}{3}}\left|d_{x y}\right\rangle \pm \sqrt{\frac{1}{3}}\left|d_{x z}\right\rangle, \\
& \left|e_{g 2}\right\rangle=-\sqrt{\frac{2}{3}}\left|d_{x^{2}-y^{2}}\right\rangle \mp \sqrt{\frac{1}{3}}\left|d_{y z}\right\rangle .
\end{aligned}
$$

Using the representation $\left|n_{a_{1 g}}, n_{e_{g 1}}, n_{e_{g 2}} ; S_{z}\right\rangle$ to denote two electrons coupled into a spin triplet with $z$ component $S^{z}=-1,0,1$ and occupying the singleparticle states Eq. (2) with occupation $n_{i}=0,1$ such that $\sum n_{i}=2$, we define new states:

$$
\begin{aligned}
\left|1, S_{z}\right\rangle & =\mp \sqrt{\frac{1}{2}}\left(\left|1,0,1 ; S_{z}\right\rangle-i\left|1,1,0 ; S_{z}\right\rangle\right), \\
\left|0, S_{z}\right\rangle & =\left|0,1,1 ; S_{z}\right\rangle, \\
\left|-1, S_{z}\right\rangle & =\mp \sqrt{\frac{1}{2}}\left(-\left|1,0,1 ; S_{z}\right\rangle-i\left|1,1,0 ; S_{z}\right\rangle\right),
\end{aligned}
$$

which are actually eigenstates of the $z$-component of the angular momentum operator $L_{z}$ projected onto the $t_{2 g}$ manifold that effectively realizes an $l=1$ representation, i.e. $L_{z}\left|M, S_{z}\right\rangle=M\left|M, S_{z}\right\rangle$, with $M=-1,0,1$. The spin-orbit coupling projected onto the basis Eq. (3) reads

$$
H_{\mathrm{SOC}}=\frac{\lambda_{\mathrm{SOC}}}{2}\left(2 S_{z} L_{z}+S^{+} L^{+}+S^{-} L^{-}\right)
$$

where $L^{ \pm}$have the same expression as for $l=1$ angular momentum operators, while the trigonal crystal field can be written as

$$
H_{\mathrm{tr}}=3 V_{\mathrm{tr}}\left(L_{z}^{2}-\frac{2}{3}\right)
$$

One can easily realize that, for $V_{\mathrm{tr}}=0$, the lowest energy state at $\lambda_{\mathrm{SOC}} \neq 0$ is five-fold degenerate, corresponding to two $d$-electrons coupled according to the Hund's rules to $S=1, L=3$ and $J=2$. Conversely, for $\lambda_{\mathrm{SOC}}=0$ but $V_{\mathrm{tr}} \neq 0$, the lowest energy state $\left|0, S_{z}\right\rangle$ is an orbitally non-degenerate spin-triplet.

However, when both parameters are finite with $\lambda_{\mathrm{SOC}} \ll V_{\mathrm{tr}}$, the lowest energy state is

$$
|0\rangle \equiv \cos \theta|0,0\rangle-\frac{\sin \theta}{\sqrt{2}}(|1,1\rangle+|-1,-1\rangle),
$$

with

$$
\tan 2 \theta=\frac{2 \sqrt{2} \lambda_{\mathrm{SOC}}}{3 V_{\mathrm{tr}}+\lambda_{\mathrm{SOC}}}
$$


TABLE II: Tight-binding parameters (in eV) obtained for corundum phase $\mathrm{V}_{2} \mathrm{O}_{3}$ by Saha-Dasgupta et al..$^{21}$ Nomenclature according to Ref. 12 .

\begin{tabular}{cccccc}
\hline \hline$\mu$ & $\rho$ & $-\alpha$ & $\beta$ & $\sigma$ & $-\tau$ \\
\hline 0.06 & -0.51 & 0.08 & -0.21 & -0.03 & -0.26 \\
\hline \hline
\end{tabular}

followed by the doublet

$$
| \pm 1\rangle \equiv \cos \phi|0, \pm 1\rangle-\sin \phi|\mp 1,0\rangle,
$$

with $\tan 2 \phi=2 \lambda_{\mathrm{SOC}} / 3 V_{\mathrm{tr}}$.

If we regard the three states $|0\rangle$ and $| \pm 1\rangle$ as the effective $S=1$ states of each isolated Vanadium, the above results show that the spin-orbit coupling generates an easy-plane anisotropy

$$
H_{*}=\Gamma_{\mathrm{tr}} S_{z}^{2} \simeq \frac{\lambda_{\mathrm{SOC}}^{2}}{3 V_{\mathrm{tr}}} S_{z}^{2} .
$$

We could proceed and consider the effects of a monoclinic distortion $V_{m} \ll V_{\text {tr }}$ that makes the $e_{g}^{\pi}$ orbitals inequivalent. ${ }^{20}$ Looking at the basis set Eq. (3), one realizes that the leading effect of this distortion corresponds to the additional operator

$$
H_{m}=V_{m}\left(L^{z} L^{y}+L^{y} L^{z}\right),
$$

whose action on the $S=1$ basis $|0\rangle$ and $| \pm 1\rangle$ above is equivalent to an additional anisotropy term

$$
\delta H_{*}=\Gamma_{m}\left(S^{z} S^{y}+S^{y} S^{z}\right),
$$

which, together with Eq. (8), might indeed justify the magnetic moment lying in the monoclinic $a-c$ plane, assumed here to be the $y-z$ plane, $29^{\circ}$ above the hexagonal basal plane.

Evidently, the above calculation is a very rough one. However it shows that one can rationalize the observed magnetic anisotropy already at the level of a single Vanadium, without invoking the "dimer" as a building block $\underline{20}$

\section{MODEL STUDIES AND DISCUSSION}

In the previous sections we have shown that the GGA+U accurately accounts for the magnetic, structural and conducting properties of $\mathrm{V}_{2} \mathrm{O}_{3}$ in its low temperature phase. This suggests the possibility of recovering similar results by model calculations within an independentparticle approximation.

An obvious starting point is a three-orbital tightbinding model for the corundum phase supplied by a local Hubbard $U$ and a Coulomb exchange $J$. To allow comparison with previous works, tight-binding parameters calculated by Saha-Dasgupta et al $\stackrel{21}{\curvearrowleft}$ in an NMTO basis set are used, which also reproduce well our GGA band structure. They are summarized in Tab. III, following the nomenclature of Ref. 12. In comparison with the parameters obtained by Refs. 12 and 30 , they are characterized by relatively small out-of-plane hopping amplitudes, $\mu$ and $\rho$, and by slightly different in-plane values, $\alpha$ and $\beta$. According to Ref. 21, the monoclinic distortion would lead to a change up to $4 \%$ of the hopping amplitudes, with the possible exception of the out-of-plane ones. We shall therefore not account for those changes and keep using the corundum structure parameters. The two-particle interaction, which includes a Hubbard $U$ and a Coulomb exchange that we assume equal to $J=U / 4$, is treated within the Hartree-Fock approximation. This amounts to consider a trial wavefunction ground state of a non-interacting Hamiltonian with the same hopping amplitudes and, in addition, with spin and orbital dependent on-site energies. We shall use an 8-site supercell, which allows to describes the "true" antiferromagnetic state, so that there are in principle 8 sets of HartreeFock on-site energies to be determined by minimizing the average total energy.

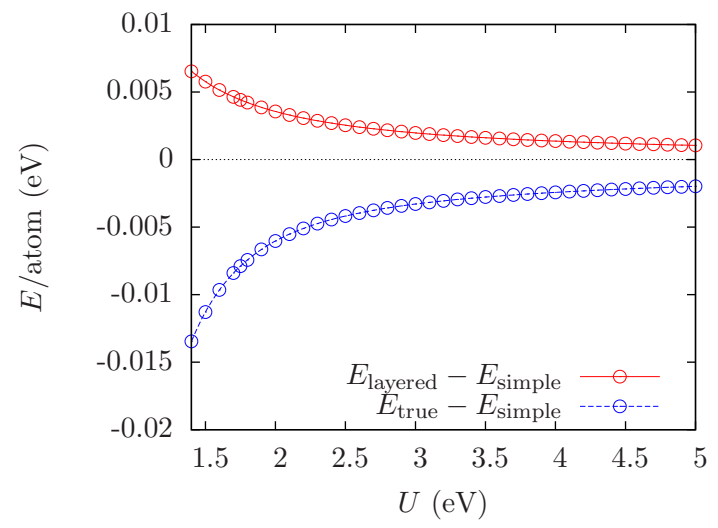

FIG. 13: (Color online) Phase stability of the insulating phases from a Hartree-Fock calculation using tight binding parameters obtained by Saha-Dasgupta et $a l^{21}$ for corundum $\mathrm{V}_{2} \mathrm{O}_{3}$.

Fig. 13 shows the Hartree-Fock total energies for each of the magnetic configurations that are obtained by a suitable choice of the initial configuration for values of $U$ above which all solutions are insulating. The comparison with GGA+U in the corundum phase is in fact not bad, although we cannot compare directly the value of $U$ used here with that in GGA+U, which already at $U=0$ includes correlation effects. Therefore it is not surprising that the "true" structure remains the lowest energy one at least up to $U=5$.

Bearing in mind the comparatively large differences of the reported values of the out-of-plane hopping, a first check of the model accuracy is to estimate the influence thereof onto the overall phase stability, which is highlighted in Fig. [14 in the extreme limit of vanishing out-ofplane hopping parameters, $\mu=\rho=0$. This check is also related to one of the main effects of the monoclinic distor- 


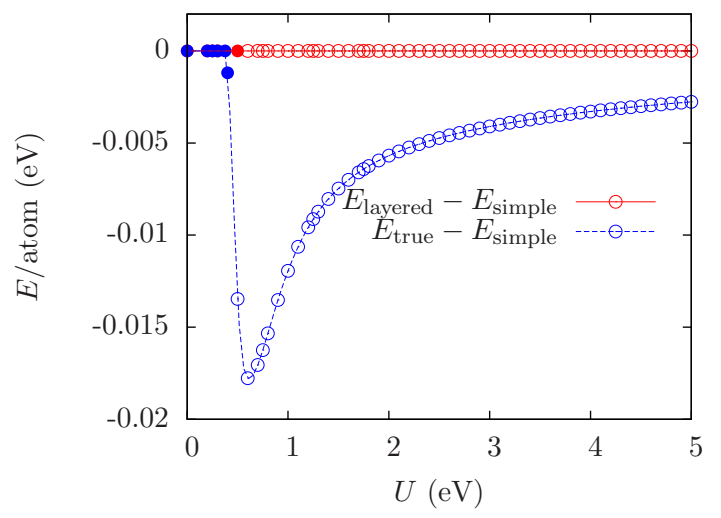

FIG. 14: (Color online) Phase stability from a Hartree-Fock calculation using tight binding parameters obtained by SahaDasgupta et $a l^{21}$ for corundum $\mathrm{V}_{2} \mathrm{O}_{3}$, but fixing all hopping perpendicular to the Vanadium planes to 0 . Closed circles indicate metallic, open circles indicate insulating solutions.

tion, which tilts and stretches the out-of-plane Vanadium dimer bonds, see Fig. [11. When $\mu=\rho=0$, the "layered" and "simple" orderings are obviously degenerate, but the "true" one is still stable up to large values of $U$, although with a smaller energy difference. This suggests that the reason for the stability of the "true" structure is primarily in the in-plane physics. The out-of-plane hopping further stabilizes this phase, at the same time destabilizing the "layered" structure compared to the "simple" one.

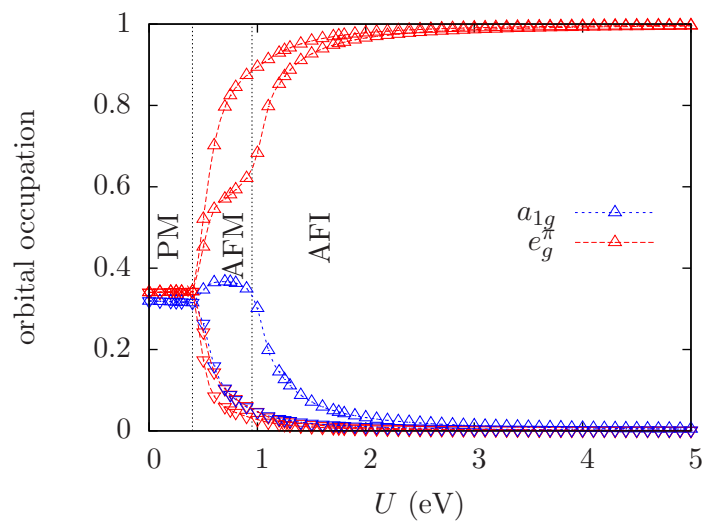

FIG. 15: (Color online) Orbital occupations in the "true" antiferromagnetic phase from Hartree-Fock as a function of $U$. Triangles pointing up indicate the majority spin channel, triangles pointing down the minority spin channel. PM, AFM and AFI stand for paramagnetic metal, antiferromagnetic metal and antiferromagnetic insulator, respectively.

The occupation numbers of the three orbitals on each Vanadium site, displayed in Fig. 15. show a very similar behavior to the GGA+U results of Fig. 10, with the exception of the small- $U$ paramagnetic metal phase. In the intermediate (unphysical) antiferromagnetic metallic regime, one can even see a slight increase of the majorityspin $a_{1 g}$ occupations, but a sharp decrease at the metalinsulator transition reveals again the scenario in which the two electrons per site occupy the $e_{g}^{\pi}$ orbitals, making the $a_{1 g}$ orbitals practically empty. Note that this orbital polarization turns out to be stronger than in GGA+U, which can be attributed to the fact that the total occupation of the Hartree-Fock model is kept fixed at 2, so that no contributions of e. g. neighboring oxygen atoms can occur. How to adapt the occupation numbers to compare with experimental findings is shortly discussed in Ref. 1.

Furthermore, the aforementioned splitting of the $e_{g}^{\pi}$ orbitals shows up also in the "true" antiferromagnetically ordered phase with hopping parameters of the corundum structure, as opposed to the "layered" and "simple" ordering. This is a further evidence that the structural distortion is intimately tight to the magnetic ordering. We highlight that such a splitting is uniform within all the eight sites of the supercell, as we also found by GGA+U. In other words, both Hartree-Fock approximation and GGA $+\mathrm{U}$ predict a ferro-orbital ordering within the $e_{\Omega}^{\pi}$ manifold, unlike the antiferro-orbital one found in Ref. [12.

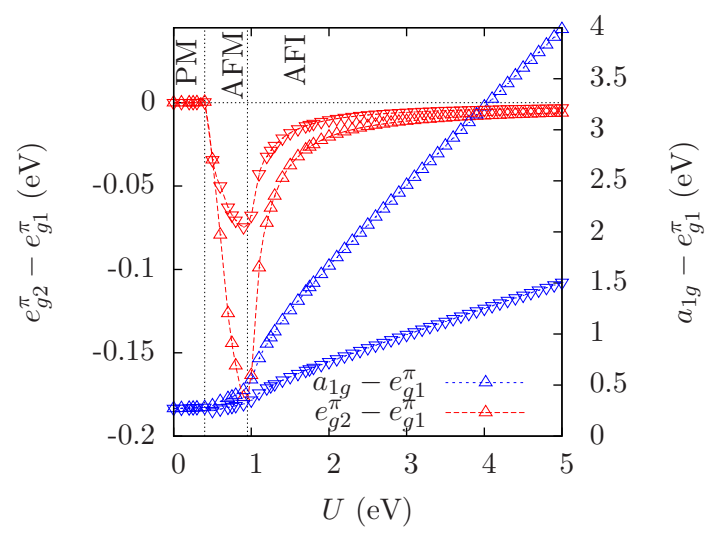

FIG. 16: (Color online) Differences of the on-site energy terms in the "true" antiferromagnetic phase from Hartree-Fock as a function of $U$. Triangles pointing up indicate the majority spin channel, triangles pointing down the minority spin channel.

The Hartree-Fock on-site energy terms, shown in Fig. 16, display the well-known large increase of the $e_{g^{-}}$ $a_{1 g}$ crystal field splitting with increasing value of $U$, especially for the majority spin, whereas all three orbitals are basically unoccupied for the minority spin. Also the splitting of the $e_{g}$ orbitals is visible, which amounts to the relatively large value of $30 \mathrm{meV}$ for the majority spin in the region of realistic parameter values. We note that the lowering of $e_{g 1}^{\pi}$ with respect to $e_{g 2}^{\pi}$ follows from our choice of a specific "true" magnetic order among the three equivalent ones allowed by the original $C_{3}$ symmetry.

In conclusion, even the Hartree-Fock approximation to a three-band Hubbard model with realistic hopping parameters reproduces the correct magnetic structure and indicates the tendency towards a spontaneous monoclinic distortion. 


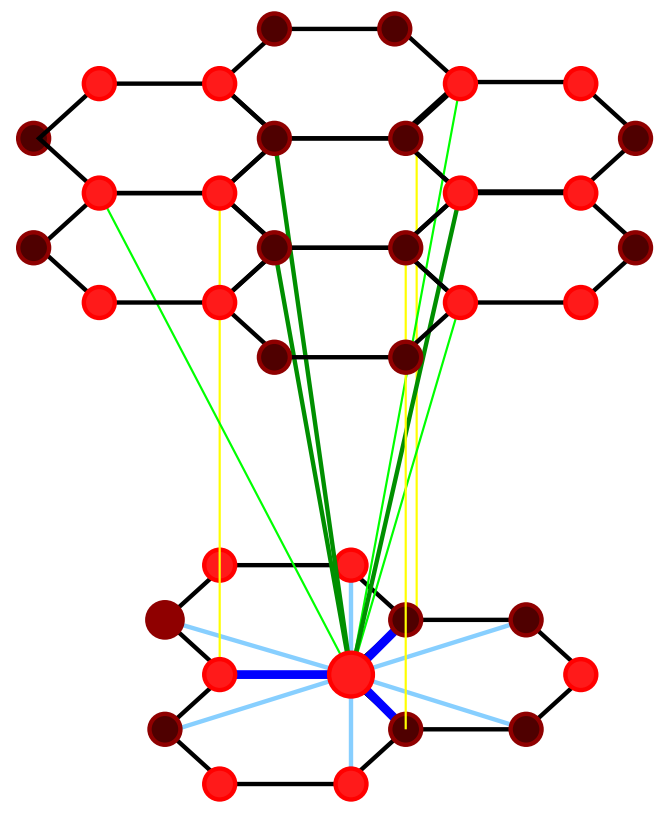

FIG. 17: (Color online) Illustration of the magnetic ordering (different spin directions as light red and dark red) and of the different relevant hopping processes from the central atom shown as a large circle. Besides the nearest neighbour $e_{g}^{\pi}-e_{g}^{\pi}$ direct hopping, dark blue, we draw the $a_{1 g}$-mediated next nearest neighbour ones: light blue in the hexagonal plane, and green between planes. Bold green lines denote that two independent paths produce the same hopping process.

\section{A. Role of the $a_{1 g}$ orbital}

Within both GGA+U and Hartee-Fock the effect of $U$ is to increase repulsion between occupied and unoccupied states, hence between majority and minority spins and between $e_{g}^{\pi}$ and $a_{1 g}$ orbitals. Therefore, at large enough $U$, the $a_{1 g}$ orbital can be discarded and one expects a two-sublattice antiferromagnetic order to prevail. This is indeed the case, see Fig. 8. It thus follows that the $a_{1 g}$ orbital must play a role to stabilise the "true" magnetic ordering for intermediate values of $U$. We argue that such a role is to provide magnetic frustration.

Let us imagine that the effective crystal field splitting, enhanced by $U$, is large enough that we can treat the hopping $\tau$, see Table II between $e_{g}^{\pi}$ and $a_{1 g}$ in perturbation theory. Let us focus on the Vanadium atom drawn as a large circle in Fig. 17. At second order, $\tau$ induces next nearest neighbour $e_{g}^{\pi}-e_{g}^{\pi}$ hopping terms, shown as light blue lines in Fig. 17, that compete against the nearest neighbour ones, drawn in dark blue. If we also take into account the large direct $a_{1 g}-a_{1 g}$ hopping along the $c$-axis, $\rho$ in Table II next nearest neighbour $e_{g}^{\pi}-e_{g}^{\pi}$ hopping processes between adjacent planes are generated, see green lines in Fig. 17, where the bold ones indicate that there are two different paths contributing to that process.

If we could use these hopping elements to derive an effective $S=1$ Heisenberg model, we would find on each plane both nearest, $J_{1}$, and next nearest, $J_{2}$, neighbour exchange constants. The phase diagram of the $S=1 / 2$ $J_{1}-J_{2}$ Heisenberg model on the honeycomb lattice is relatively well known ${ }^{40}-42$ The two-sublattice antiferromagnet is stable for $J_{2} \lesssim 0.2 J_{1}$. For $0.2 \lesssim J_{2} / J_{1} \lesssim 0.4$, there seems to be no magnetic order. Finally, for $J_{2} \gtrsim 0.4 J_{1}$ the magnetic order is exactly the "true" antiferromagnet, shown in Fig. 17. We cannot exclude that the larger spin $S=1$ and the coupling between the planes could stabilise in our case the "true" antiferromagnet even in the formerly disordered region $0.2 \lesssim J_{2} / J_{1} \lesssim 0.4$.

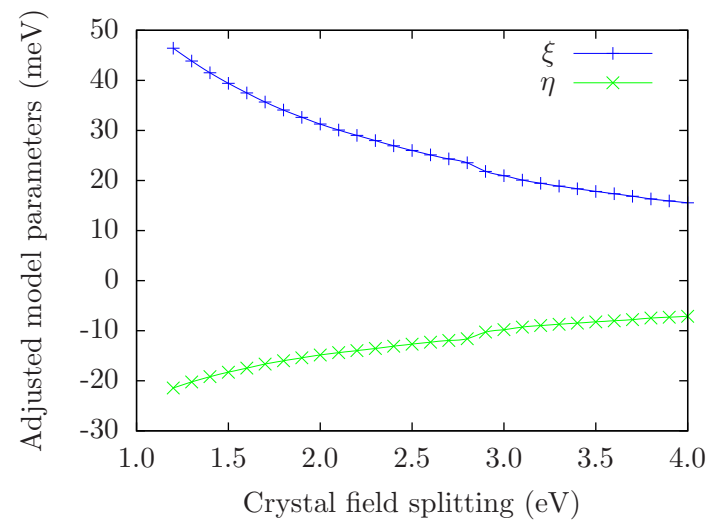

FIG. 18: (Color online) Fitted next-nearest neighbour inplane hopping parameters $\xi$ and $\eta$. See text for details.

In order to estimate the amount of frustration brought by the $a_{1 g}$-mediated next-nearest neighbour hopping, we have calculated the band structure in the corundum phase as function of an artificial crystal field spitting. When the crystal field is large enough, $\gtrsim 1.2 \mathrm{eV}$, the upper $a_{1 g}$-derived bands are well separated from the lower $e_{g}^{\pi}$-derived ones, hence we can fit the latter by a simple tight-binding model with only $e_{g}^{\pi}-e_{g}^{\pi}$ nearest and next-nearest neighbour hopping parameters. The nearest neighbour ones are assumed to be those in Table II. By the three-fold rotational symmetry, we just need two fitting next-nearest neighbour hopping parameters within the hexagonal planes, which we call $\xi$ and $\eta$ and plot in Figure [18. As expected, $\xi$ and $\eta$ decrease in absolute value as the crystal field increases. However, for not too large crystal field splitting, $\xi$ and $\eta$ have the same order of magnitude as the nearest neighbour hopping parameters $\alpha$ and $\beta$, see Table $\amalg$.

The above calculation is very rough; the effective crystal field is not so large that $e_{g}^{\pi}$ and $a_{1 g}$ bands are separated, and $U$ not big enough to justify a mapping onto an Heisenberg model. However we believe that the overall scenario is correct: the $a_{1 g}$ orbital, although pushed by relatively strong correlations above Fermi in the insulating phase, as first noted by DMFT in Ref. 1, still heavily contributes to stabilise the "true" magnetic structure. All these features, including the monoclinic distortion and the magnetic anisotropy, are well captured by an independent particle scheme as GGA+U. 


\section{Acknowledgments}

We wish to thank S. Shahab Naghavi, Ryan Tyler Requist, Matteo Sandri, Andrea dal Corso and Erio Tosatti for very helpful discussions. This work has been supported by the European Union, Seventh Framework Programme, under the project GO FAST, grant agreement no. 280555 .
1 A. I. Poteryaev, J. M. Tomczak, S. Biermann, A. Georges, A. I. Lichtenstein, A. N. Rubtsov, T. Saha-Dasgupta, and O. K. Andersen, Phys. Rev. B 76, 085127 (2007).

2 D. B. McWhan, J. P. Remeika, T. M. Rice, W. F. Brinkman, J. P. Maita, and A. Menth, Phys. Rev. Lett. 27, 941 (1971).

3 D. B. McWhan, A. Menth, J. P. Remeika, T. M. Rice, and W. F. Brinkman, Phys. Rev. B 7, 1920 (1973).

4 M. Foëx, Comptes rendus hebdomadaires des séances de l'Académie des sciences 223, 1126 (1946).

5 R. M. Moon, Phys. Rev. Lett. 25, 527 (1970).

${ }^{6}$ D. B. McWhan and J. P. Remeika, Phys. Rev. B 2, 3734 (1970).

7 P. D. Dernier and M. Marezio, Phys. Rev. B 2, 3771 (1970).

8 D. Grieger, C. Piefke, O. E. Peil, and F. Lechermann, Phys. Rev. B 86, 155121 (2012).

9 S. Y. Ezhov, V. I. Anisimov, D. I. Khomskii, and G. A. Sawatzky, Phys. Rev. Lett. 83, 4136 (1999).

10 C. Tenailleau, E. Suard, J. Rodriguez-Carvajal, A. Gibaud, and P. Lacorre, Journal of Solid State Chemistry 174, 431 (2003).

11 P. Rozier, A. Ratuszna, and J. Galy, Zeitschrift für anorganische und allgemeine Chemie 628, 1236 (2002).

12 C. Castellani, C. R. Natoli, and J. Ranninger, Phys. Rev. B 18, 4967 (1978).

13 C. Castellani, C. R. Natoli, and J. Ranninger, Phys. Rev. B 18, 4945 (1978).

14 C. Castellani, C. R. Natoli, and J. Ranninger, Phys. Rev. B 18, 5001 (1978).

15 K. I. Kugel' and D. I. Khomskii, Sov. Phys. Usp. 25, 231 (1982).

16 J.-H. Park, L. H. Tjeng, A. Tanaka, J. W. Allen, C. T. Chen, P. Metcalf, J. M. Honig, F. M. F. de Groot, and G. A. Sawatzky, Phys. Rev. B 61, 11506 (2000).

17 L. Paolasini, C. Vettier, F. de Bergevin, F. Yakhou, D. Mannix, A. Stunault, W. Neubeck, M. Altarelli, M. Fabrizio, P. A. Metcalf, et al., Phys. Rev. Lett. 82, 4719 (1999)

18 F. Mila, R. Shiina, F.-C. Zhang, A. Joshi, M. Ma, V. Anisimov, and T. M. Rice, Phys. Rev. Lett. 85, 1714 (2000).

19 S. Di Matteo, N. B. Perkins, and C. R. Natoli, Journal of Physics: Condensed Matter 14, L37 (2002).

20 A. Tanaka, Journal of the Physical Society of Japan 71, 1091 (2002).

21 T. Saha-Dasgupta, O. K. Andersen, J. Nuss, A. I. Poteryaev, A. Georges, and A. I. Lichtenstein,
arXiv:0907.2841 (2009).

22 I. S. Elfimov, T. Saha-Dasgupta, and M. A. Korotin, Phys. Rev. B 68, 113105 (2003).

23 N. B. Perkins, S. Di Matteo, and C. R. Natoli, Phys. Rev. B 80, 165106 (2009).

24 S. Di Matteo, Physica Scripta 71, CC1 (2005).

${ }^{25}$ K. Held, G. Keller, V. Eyert, D. Vollhardt, and V. I. Anisimov, Phys. Rev. Lett. 86, 5345 (2001).

26 J. P. Perdew, K. Burke, and M. Ernzerhof, Phys. Rev. Lett. 77, 3865 (1996).

27 P. Giannozzi, S. Baroni, N. Bonini, M. Calandra, R. Car, C. Cavazzoni, D. Ceresoli, G. L. Chiarotti, M. Cococcioni, I. Dabo, et al., Journal of Physics: Condensed Matter 21, 395502 (2009), URL http://www.quantum-espresso.org

28 P. D. Dernier, J. Phys. Chem. Solids 31, 2569 (1970).

29 Y. Ding, C.-C. Chen, Q. Zeng, H.-S. Kim, M. J. Han, M. Balasubramanian, R. Gordon, F. Li, L. Bai, D. Popov, et al., Phys. Rev. Lett. 112, 056401 (2014).

30 L. F. Mattheiss, J. Phys.: Condens. Matter 6, 6477 (1994).

31 V. Eyert, U. Schwingenschlögl, and U. Eckern, EPL (Europhysics Letters) 70, 782 (2005).

32 D. Grieger and F. Lechermann, Phys. Rev. B 90, 115115 (2014).

33 M. Cococcioni and S. de Gironcoli, Phys. Rev. B 71, 035105 (2005).

34 A. Abragam and B. Bleaney, Introduction to ligand field theory (Clarendon Press - Oxford, 1970), pp. 377-378 and 426-429.

35 G. M. Cole and B. B. Garrett, Inorganic Chemistry 9, 1898 (1970).

36 O. Tchernyshyov, Phys. Rev. Lett. 93, 157206 (2004).

37 N. B. Perkins and O. Sikora, Phys. Rev. B 76, 214434 (2007).

38 A. Dal Corso and A. Mosca Conte, Phys. Rev. B 71, 115106 (2005).

39 A. I. Liechtenstein, V. I. Anisimov, and J. Zaanen, Phys. Rev. B 52, R5467 (1995).

40 A. Albuquerque, D. Schwandt, B. Hetényi, S. Capponi, M. Mambrini, and A. Läuchli, Phys. Rev. B 84, 024406 (2011).

41 J. Fouet, P. Sindzingre, and C. Lhuillier, The European Physical Journal B - Condensed Matter and Complex Systems 20, 241 (2001).

42 F. Mezzacapo and M. Boninsegni, Phys. Rev. B 85, 060402 (2012). 